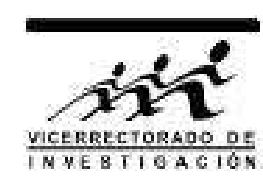

\title{
Reconstrucción de imágenes para tomografía de pozo a pozo con el método de Levenberg Marquardt
}

\author{
Juan Chambi Chambi, Raul Carita Montero y Mariella Berrocal Tito* \\ Facultad de Ciencias Físicas, Universidad Nacional Mayor de San Marcos, \\ Ciudad Universitaria, Av. Venezuela s/n, Lima 14, Perú
}

Recibido 28 marzo 2014 - Aceptado 15 mayo 2014

\begin{abstract}
La tomografía de pozo a pozo es uno de los métodos sísmicos más usados para la determinación de la estructura de los subsuelos. Este método consiste en colocar en un pozo las fuentes que generan las ondas sísmicas, y en otro pozo los receptores de las ondas. La imagen reconstruida en la tomografía de pozo a pozo es obtenida al considerar el parámetro denominado lentitud, que es la inversa de la velocidad con la que viajan las ondas sísmicas, la lentitud $S$ varía según la estructura geológica del medio de propagación. Considerando ondas directas que viajan de un pozo a otro, realizamos una aproximación lineal para obtener $S$ como $M S=T$, donde $M$ es una matriz y está relacionado con la discretización del dominio, $S$ es el vector formado por la lentitud de cada elemento discretizado del dominio y $T$ es el tiempo de llegada de la primera onda desde la fuente al detector.

Para la evaluación de los algoritmos diseñamos estructuras de subsuelos, de los cuales obtenemos los datos de los detectores. Para obtener datos más realísticos, se les agrega un ruido aleatorio hasta $3.0 \%$. Con esos datos se procede a reconstruir las propiedades del suelo.
\end{abstract}

Palabras claves: Problema inverso, tomografía pozo a pozo, método Levenberg Marquardt.

\section{Images reconstruction for well to well tomography with Levenberg Marquardt method}

The Cross-Borehole-Seismic Tomography is a seismic method most used for the determination of subsurface structure of the land. This method involves placing in a borehole the seismic wave generating sources, and in another borehole the receiver as waves detector.

The tomographic image reconstructed from Cross-Borehole-Seismic Tomography is obtained by considering a parameter called slowness which is reverse speed with which the seismic waves travel, slowness $S$ varies depending on the geological structure. We consider direct waves traveling from one borehole to another and we obtain an approximation of the following linear system as $M S=T$, where $M$ is a matrix and is related to the discretization domain, $S$ is the vector formed by the slowness of each discretized domain element and $T$ is the arrival time of the first wave coming from source to detector. For the evaluation of the algorithms we design underground structures, which get the data from the detectors.

To obtain more realistic data, we added random noise to $3.0 \%$. With these data we proceed to rebuild the soil properties.

Keywords: Inverse problem, Cross-Borehole-Seismic Tomography, Levenberg Marquardt method.

Cuando se produce un sismo o una explosión, se generan diversos tipos de ondas que se propagan, aproximadamente, como rayos en todas direcciones, atravesando la tierra hasta llegar a la estación sísmica o un receptor que los registra. La trayectoria que sigue, y el tiempo que transcurre desde la generación de la onda hasta su registro, depende de la estructura del medio atravesado. Entonces se deduce que el registro de las ondas contiene informa- ción de las propiedades físicas del interior de la Tierra, muestreada a lo largo de la trayectoria del rayo desde la fuente hasta el receptor, permitiendo mediante su estudio, analizar la estructura atravesada [1,2].

El estudio por tomografía sísmica es realizado con dos clases de datos, los tiempos de recorrido y los formatos de la onda. La tomografía por tiempo de recorrido posee una resolución mucho menor pero es mucho más robusta

*mabet99@yahoo.com 
desde el punto de vista computacional y es fácil de implementar respecto a la tomografía de formatos de onda. En el presente trabajo, la magnitud física que estudiamos en el interior de la tierra es la velocidad de propagación de las ondas $P$, que se calculan a partir de los tiempos de recorrido de estas ondas desde la fuente hasta el receptor que son calculables si conocemos la posición de la fuente, el tiempo origen y el instante en que la onda llega al receptor [3].

El método de la tomografía sísmica de pozo a pozo se basa en la hipótesis de que la diferencia de tiempos puede interpretarse en términos de estructuras anómalas de la velocidad con respecto al modelo del terreno supuesto. Este método se emplea rutinariamente en la detección de reservorios petrolíferos, terrestres y marítimos, de grandes estructuras geológicas como plegamientos montañosos, de zonas de subducción, de yacimientos minerales, de domos salinos, entre otros [4]. La tomografía sísmica pozo a pozo nos permite la reconstrucción de una imagen del subsuelo que se utiliza para conocer los detalles de un yacimiento petrolífero o minero en la fase de exploración o explotación. También es útil para ubicar anomalías, como la existencia de cavidades vacías o llenas de fluídos o condensados o enormes macizos rocosos [5].

En el presente trabajo, diseñamos un experimento de tomografía de pozo a pozo y con los datos desarrollamos un tratamiento de imágenes usando el método de Levenberg Marquardt para identificar los detalles del medio en que se propaga las ondas acústicas. Desarrollamos algunos ejemplos de los métodos utilizados. Las secciones del artículo presentan inicialmente el detalle teórico de los métodos, luego la implementación de los algoritmos, la obtención de los datos y la discusión de los mismos y finalmente exponemos algunas conclusiones del trabajo.

\section{Teoría}

La tomografía pozo a pozo consiste en obtener datos de tiempo de recorrido de las ondas acústicas emitidas por una fuente localizada en uno de los dos pozos y captada por receptores en el otro pozo. Utilizando estos datos se reconstruye la imagen del subsuelo, con los diferentes caminos que recorren las ondas tal como se muestra en la Figura [1]

La región en estudio es dividida en pequeños pixeles en el caso bidimensional y en pequeños voxeles para el caso tridimensional.

Para estudiar rigurosamente la propagación de las ondas sísmicas se recurre a estudiar las ecuaciones de la elastodinámica de los medios contínuos. El principio de Fermat sobre la propagación de los rayos de luz, afirma que la trayectoria real entre dos puntos tomada por un haz de luz es aquella que es recorrida en el tiempo mínimo [6].

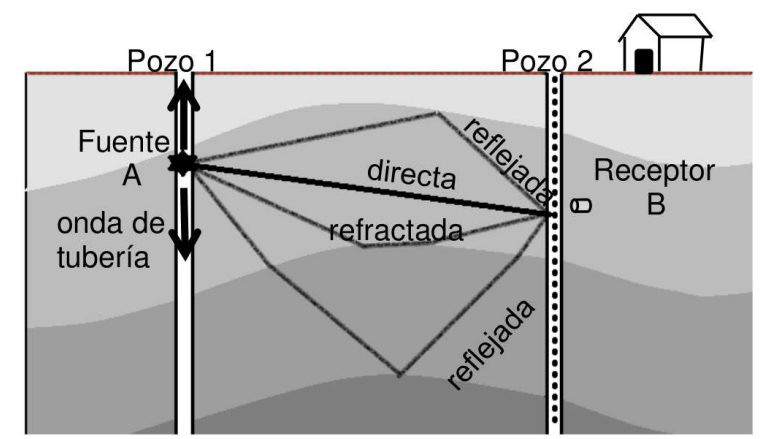

Figura 1: Esquema de la tomografía pozo a pozo y los diferentes recorridos de las ondas acústicas.

Para aplicar el principio de Fermat a las ondas sísmicas necesitamos saber si estas ondas pueden ser consideradas como ondas planas. Las ondas sísmicas si son emitidas por una fuente puntual, los frentes de ondas cercanas a la emisión son frentes de ondas esféricos pero a medida que la onda se va alejando de la fuente el frente de onda, deviene a ser plano. Entonces para distancias lejanas del origen de las ondas sísmicas hablamos de la propagación de ondas planas, en este caso de rayos sísmicos [6].

Para calcular el tiempo de llegada de un rayo sísmico desde un punto lejano al receptor, introducimos el concepto de lentitud, $S(x)$, que es la inversa de la velocidad, $v(x)$.

El tiempo de llegada de una señal desde la fuente emitida $A$ hacia el receptor $B$ a través de un posible camino está dado por

$$
t_{A B}=\int_{A}^{B} S(x) d \ell=\int_{A}^{B} \frac{a}{v(x)} d \ell
$$

donde $S$ es una distribución contínua de lentitudes $s(x)$ y $d \ell$ indica el diferencial de camino de $A$ hacia $B$.

Es necesario resaltar que los caminos por los que viajan la onda no son necesariamente líneas rectas, lo que torna a la ecuación, que modela la tomografía pozo-pozo, no lineal. Este hecho se debe a que el desplazamiento del frente de onda es una curva que depende de la lentitud. Las ondas utilizadas para la colección de datos en la tomografía pozo a pozo, está sujeta a la ley de Snell y el índice de refracción está lejos de ser constante por ello los recorridos no necesariamente son líneas rectas.

Cuando una onda sísmica encuentra un cambio en las propiedades elásticas del material, como es el caso de una interface entre dos capas geológicas, una parte de la energía continúa en el mismo medio, onda incidente, una parte se refleja, ondas reflejadas y el resto se transmite al otro medio, ondas refractadas, con cambios en la dirección de propagación, en la velocidad y en el modo de vibración, Figura 2]

Las leyes de la reflexión y la refracción se derivan por el principio de Huygens cuando se considera un frente de onda que incide sobre una interface plana [7]. La ley de 
Snell relaciona los índices de refracción y los ángulos de incidencia y refracción de una onda que atraviesa dos medios de diferentes lentitudes o índices de refracción. Como el índice de refracción es dado por $n=c / v$, donde $c$ es la velocidad de la luz en el vacío o la onda en un medio homogéneo. Luego la ley de Snell es definida como

$$
s_{1} \sin \theta_{1}=s_{2} \sin \theta_{2}
$$

donde $\theta_{1}$ y $\theta_{2}$ son los ángulos del rayo de incidencia y del rayo refractado a la normal al plano que separa las dos regiones, respectivamente. $\mathrm{Y} s_{1}$ y $s_{2}$ son las lentitudes en los medios 1 y 2 , respectivamente.

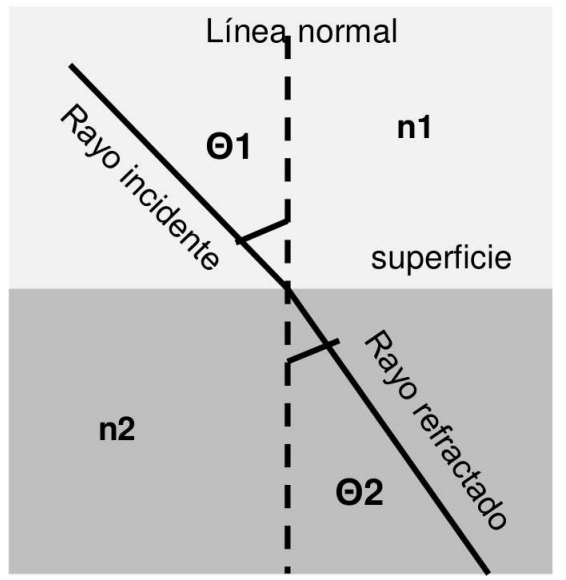

Figura 2: Conversión de una onda incidente $P$. Las ondas sísmicas que viajan por el subsuelo se reflejan y se refractan siguiendo la ley de Snell.

La cantidad de energía de las ondas incidentes se reparte entre las ondas reflejadas, las refractadas y la absorción natural del terreno [7]. La ley de Snell proporciona información sobre las trayectorias de los rayos, los tiempos de llegada y la posición de los refractores, pero no proporciona información alguna sobre las amplitudes de las ondas [7].

Del principio de Fermat y de la ley de Snell obtenemos que las ondas que llega primero desde la fuente al receptor son las que hacen el camino más recto.

\section{El problema directo y el problema inverso}

Cuando se determina el tiempo de llegada de una onda desde la fuente al receptor usando la Ec.(1), conociendo la posición de la fuente y del detector, así como las características del medio, es lo que se denomina un problema directo. A partir de las medidas de los detectores la determinación de las lentitudes de la trayectoria recorrida sobre el medio desde la fuente al detector, es lo que se denomina un problema inverso.

Un problema directo, es representado a través de una ecuación que cumple tres condiciones, tiene solución, la solución es única, y tiene estabilidad en relación a la variación de los datos de entrada. Un problema inverso, es un problema que no cumple con las condiciones de un problema directo. Puede tener muchas soluciones, por lo que es necesario aplicar diferentes técnicas de problemas inversos para encontrar una solución que sea la más óptima, y tenga una estabilidad relativa a los datos de entrada.

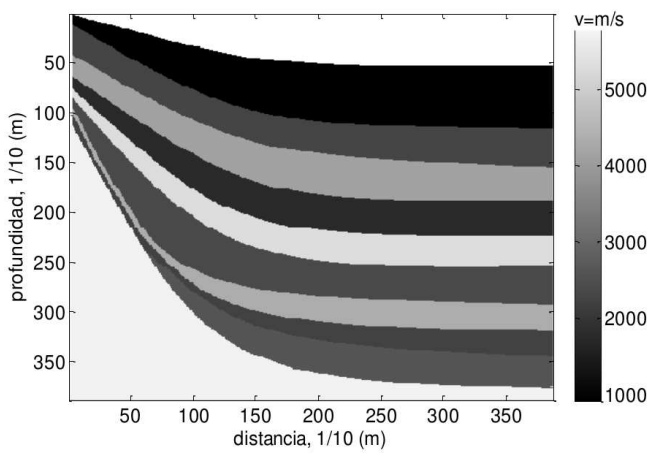

Figura 3: Muestra de un tomograma sísmico.

Existen una variedad de técnicas de problemas inversos que son empleados en la prospección sísmica, para determinar los perfiles de la región en estudio, siendo el objetivo obtener un tomograma sísmico, como se observa en la Figura 3 donde cada color y cada tono, nos indica una determinada velocidad o lentitud que representa a un particular medio. Se puede decir que el tomograma sísmico representa un campo de velocidades. Cada velocidad está relacionada a un tipo de material, como se describe en la Figura 4 . Con la solución obtenida, el tomograma, los geólogos y geofísicos infieren el tipo de material existente en el subsuelo [7]. Para la adquisición de los datos para el problema inverso son utilizadas como fuentes de energía sísmica la dinamita o un vibrador de tierra.

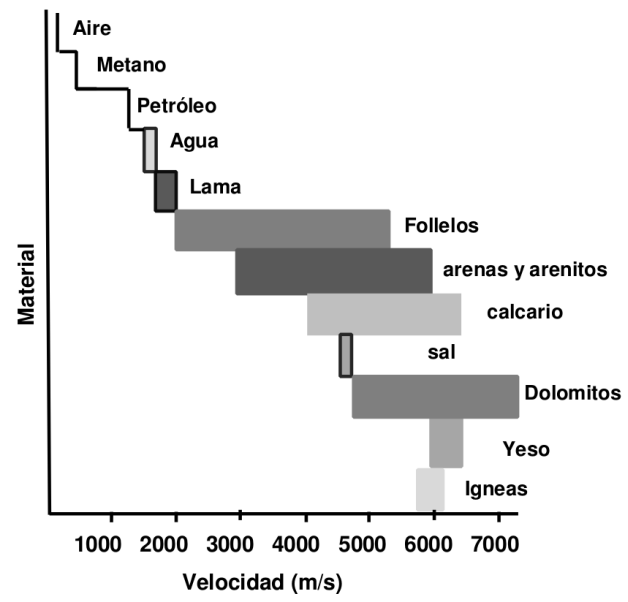

Figura 4: Se muestran algunos materiales en función de las velocidades permitidas para la propagación de las ondas sísmicas [3].

Para una región bidimensional, el medio es particionado en pequeños pixeles. Las trayectorias de recorrido de la 
señal cruzan determinados pixeles. Esta información de las longitudes que se cruzan en cada pixel por cada trayectoria de la señal es representado por una matriz $M$. Para la construcción de la matriz $M$ se considera un dominio rectangular de un tamaño dado que discretizamos en pixeles $a_{e}$ tal como se muestra en la Figura 5 Luego enumeramos cada pixel y definimos para cada pixel una lentitud $s_{e}$, el cual también es enumerado como se muestra en la misma Figura 5. Si E es el número total de pixeles enumerados, las lentitudes del medio son representados a través de un vector $\mathbf{S}$, de modo que cada elemento de $\mathbf{S}$, representado por $s_{e}$ indica la lentitud de la propagación en un pixel $\mathrm{a}_{e}$.

Definimos las posiciones de las fuentes y de los receptores de modo que $\mathbf{F}$ es el número total de fuentes y $\mathbf{D}$ es el número total de detectores, luego trazamos las trayectorias que une las fuentes a los receptores, lo que produce $K=F \times D$ trayectorias.

\begin{tabular}{|l|l|l|l|}
\hline$a_{1}$ & $a_{2}$ & $a_{3}$ & $a_{4}$ \\
\hline$a_{5}$ & $a_{6}$ & $a_{7}$ & $a_{8}$ \\
\hline$a_{9}$ & $a_{10}$ & $a_{12}$ & $a_{11}$ \\
\hline$a_{13}$ & $a_{14}$ & $a_{15}$ & $a_{16}$ \\
\hline
\end{tabular}

\begin{tabular}{|l|l|l|l|}
\hline $\mathrm{s}_{1}$ & $\mathrm{~s}_{2}$ & $\mathrm{~s}_{3}$ & $\mathrm{~s}_{4}$ \\
\hline $\mathrm{s}_{5}$ & $\mathrm{~s}_{6}$ & $\mathrm{~s}_{7}$ & $\mathrm{~s}_{8}$ \\
\hline $\mathrm{s}_{9}$ & $\mathrm{~s}_{10}$ & $\mathrm{~s}_{12}$ & $\mathrm{~s}_{11}$ \\
\hline $\mathrm{s}_{13}$ & $\mathrm{~s}_{14}$ & $\mathrm{~s}_{15}$ & $\mathrm{~s}_{16}$
\end{tabular}$\quad S=\left[\begin{array}{c}s_{1} \\
s_{2} \\
s_{3} \\
: \\
s_{14} \\
s_{15} \\
s_{16}\end{array}\right]$

Figura 5: Se muestra un ejemplo del proceso de la discretización del dominio de pixeles $a_{e}$, asociados a los elementos $s_{e}$ del vector lentitud.

La Figura 6 muestra los trazados de los caminos posibles entre las fuentes y los receptores, que es justificado por una aproximación lineal de acuerdo al Principio de Fermat que ignora la dependencia de los caminos con respecto a la distribución de lentitudes, el dominio es discretizado en $12 \times 10$ celdas, considerando doce las fuentes situadas en la frontera izquierda y doce los detectores situados en el lado derecho, las líneas azules son las trayectorias recorridas por por los frentes de onda del sonido.

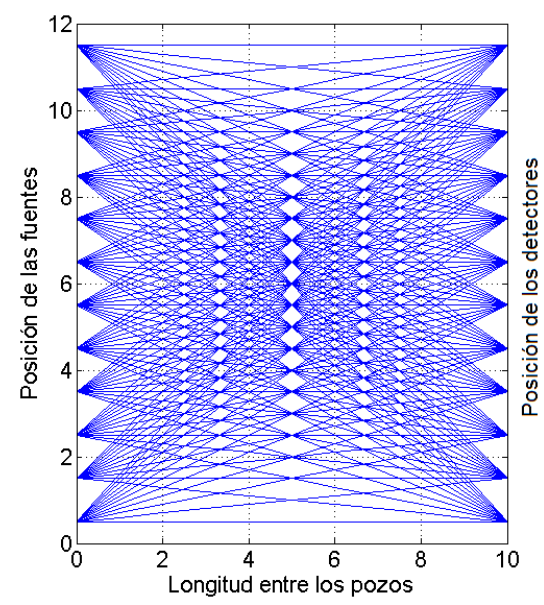

Figura 6: Trayectorias para un dominio de $10 \times 12$ pixels, con 12 fuentes y 12 detetectores.
Cada trayectoria atraviesa un determinado número de pixeles, cada longitud de la trayectoria recorrida en cada pixel es representada por $l_{e k}$, donde $e$ indica el pixel y $k$ indica la trayectoria. Para un recorrido $k$, el tiempo medido en ese pixel de acuerdo a la Ec 1, está dado por

$$
t_{k}=l_{e k} s_{e},
$$

donde $s_{e}$ es el inverso de la velocidad en el $e$-ésimo pixel, suponiendo que la velocidad es constante en cada pixel. Consideremos una trayectoria que atraviesa varios pixeles desde una fuente hasta un detector, de izquierda a derecha, como se muestra en la Figura 7 y está dada por $L_{k}=\sum l_{e k}$, produciendo un tiempo,

$$
\begin{aligned}
T_{k}=\sum_{e} & l_{e k} s_{e}=l_{9 k} s_{9}+ \\
& l_{10 k} s_{10}+l_{6 k} s_{6}+l_{7 k} s_{7}+l_{3 k} s_{3}+l_{4 k} s_{4}
\end{aligned}
$$

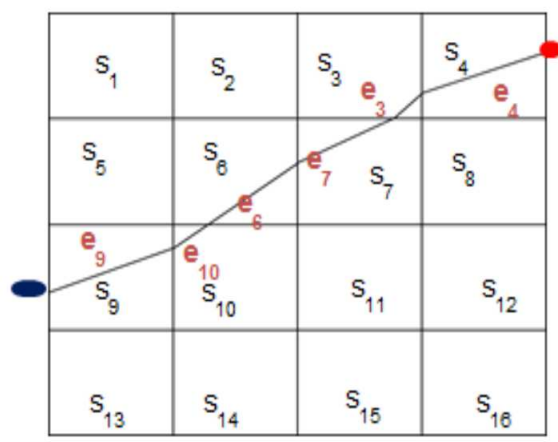

Figura 7: Se muestra una trayectoria entre una fuente (lado izquierdo) y un detector (lado derecho) cuyo tiempo de recorrido es determinado por la Ec. (4).

Por tanto, la matriz $M$ tiene $K \times E$ dimensiones, donde cada fila está relacionada a una trayectoria y cada columna a un pixel que forma el dominio espacial,

$$
M=\left(\begin{array}{ccccc}
l_{11} & \ldots & l_{1 e} & \ldots & l_{1 E} \\
\vdots & & \vdots & & \vdots \\
l_{k 1} & \ldots & l_{k e} & \ldots & l_{k E} \\
\vdots & & \vdots & & \vdots \\
l_{K 1} & \ldots & l_{K e} & \ldots & l_{K E}
\end{array}\right)
$$

asimismo, el sub-índice de la columna indica el número de pixel y el sub-índice de la fila el número de la trayectoria.

Los tiempos para cada trayectoria, desde las fuentes hasta los detectores, son los componentes del vector $T$, donde el sub-índice de cada elemento de $T$ indica el tiempo recorrido de una trayectoria. Este vector $T$ se determina como

$$
M S=T
$$




$$
\left(\begin{array}{ccccc}
l_{11} & \ldots & l_{1 e} & \ldots & l_{1 E} \\
\vdots & & \vdots & & \vdots \\
l_{k 1} & \ldots & l_{k e} & \ldots & l_{k E} \\
\vdots & & \vdots & & \vdots \\
l_{K 1} & \ldots & l_{K e} & \ldots & l_{K E}
\end{array}\right)\left(\begin{array}{c}
s_{1} \\
\vdots \\
s_{e} \\
\vdots \\
s_{E}
\end{array}\right)=\left(\begin{array}{c}
t_{1} \\
\vdots \\
t_{k} \\
\vdots \\
t_{K}
\end{array}\right)
$$

Resumiendo, el problema directo es calcular $T$, y la tomografía de pozo a pozo es un problema inverso que trata de encontrar $S$ a partir de la ecuación 6 .

La matriz $M$ es una matriz no cuadrada, escasa (del inglés sparse), con muchos elementos iguales a cero, por lo que es necesario emplear algunas técnicas para obtener $S$ que se discuten a continuación.

El método más trivial es la inversión matricial debido a que $M$ es una matriz cuadrada ésta se puede invertir y obtener de forma directa el vector de lentitudes. El segundo método es el de mínimos cuadrados basado en el algoritmo

$$
S=\left(M^{T} M\right)^{-1}\left(M^{T} T\right),
$$

donde $M^{T}$ es la transpuesta de la matriz $M$.

\section{El método de Levenberg Marquardt}

La Figura 7 considera un problema de 4 fuentes y 4 detectores, de modo que el número total de pixeles es $E=4 \times 4=16$ y el número de trayectorias también es $K=4 \times 4=16$. Por tanto, la dimensión de $M$ es $16 \times 16$. En la Figura 7] se observa también que la trayectoria delineada solo cruza 6 pixeles de los 16, luego en la matriz $M$ la trayectoria es representada por una fila de 16 elementos, de los cuales solo 6 son diferentes de cero. Algo similar se va a observar en las otras filas para otras trayectorias. Por ese motivo la matriz $M$ es denominada una matriz escasa (sparse), por estar formada mayormente por elementos iguales a cero.

La probabilidad de que $M^{T} M$ tenga una inversa, depende de que la diagonal sea dominante. Es por ello que en el método de Levenberg Marquardt se trata de dar un peso a la diagonal de la matriz $M^{T} M$, para aumentar la probabilidad de tener la matriz inversa. El algoritmo Levenberg Marquandt para obtener $S$ está dado por

$$
S=\left(M^{T} M-\lambda I\right)^{-1}\left(M^{T} T\right)
$$

donde $\lambda$ es un parámetro de amortiguamiento, e $I$ es la matriz identidad.

Existen otros métodos para determinar el problema inverso, estos son los métodos iterativos, estos consisten en aproximarse a una solución $S^{u+1}$ desde una solución inicial dada, $S^{0}$, determinando previamente en forma iterativa $S^{u}$. El superíndice en el vector, $S$, indica el número de repeticiones del proceso iterativo. Sea $\tau$ el vector formado por los tiempos de las trayectorias medidos experimentalmente, luego se asume un vector $S$ de entrada para la primera iteración y se define la funcional objetivo dado por

$$
F(S)=\frac{1}{2}\|M S-\tau\|^{2},
$$

se define el gradiente de $F(S)$ como

$$
\nabla F(S)=M^{T} M S-\tau
$$

y la Hessiana como la matriz cuadrada dado por

$$
\nabla^{2} F(S)=M^{T} M \text {. }
$$

Si $F(S)=0$, entonces el gradiente también es cero. El método de Newton consiste en determinar

$$
\nabla F(S)=0 \text {. }
$$

Para ello se define una función $G(S)=\nabla F(S)$, de modo que si $G(S)=0$, para un $S$, éste es la solución de nuestro problema.

Consideremos conocido los valores de $S^{u}$, luego también conocemos $G\left(S^{u}\right)$. Empleando la serie de Taylor podemos calcular un $G\left(S^{u+1}\right)$ como

$$
G\left(S^{u+1}\right)=G\left(S^{u}\right)+\nabla\left(G\left(S^{u}\right)\right)\left(S^{u+1}-S^{u}\right) .
$$

Luego hacemos $G\left(S^{u+1}\right)=0$ y $S^{u+1}$ será la solución, calculada por

$$
S^{u+1}=S^{u}+\frac{\nabla F\left(S^{u}\right)}{\nabla^{2} F\left(S^{u}\right)} .
$$

Al aplicar el método de Newton, debemos garantizar en la Ec.(14) que la inversa de la matriz $\nabla^{2} F\left(S^{u}\right)$ sea diferente de cero, para ello agregamos un peso $\lambda$, denominado factor de amortiguamiento a la diagonal de modo que reescribimos el algoritmo dado por la Ec.(14) como

$$
S^{u+1}=S^{u}+\frac{\nabla F\left(S^{u}\right)}{\nabla^{2} F\left(S^{u}\right)+\lambda I}
$$

donde $\lambda \in[0,1]$. A este proceso se le denomina el método de Levenberg Marquardt iterativo.

\section{Resultados}

Realizamos la reconstrucción de las imágenes para cuatro casos cuyos dominios espaciales en $x$ están dados por $30,40,50$ y 60 pixeles y en $y$ solo 60 pixeles. El número de fuentes y detectores en los cuatro casos son iguales a 60 , mientras que el número de ecuaciones a resolver son 3600 y el número de incógnitas son 1800, 2400, 3000 y 3600 , respectivamente, para los cuatro casos. Ver Tabla 1.

Buscando un número que nos indique la calidad en las estimaciones de las incógnitas en cada caso, definimos el error porcentual como

$$
E P=100 \% \times \sqrt{\sum_{r=1}^{E}\left(\frac{S_{e}^{\text {exac }}-S_{e}^{\text {calc }}}{S_{e}^{\text {exac }}}\right)^{2}},
$$


donde las lentitudes exactas son las lentitudes definidas en las imágenes originales, las lentitudes calculadas son los que son determinados del cálculo usando las las medidas de los detectores. Las medidas de los detectores son obtenidos del problema directo, sin embargo, para ser más realístico este trabajo se introdujo ruido aleatorio a las medidas de los detectores obtenido por el problema directo, empleando la expresión

$$
t_{i}=t_{i}^{\text {exac }}(1+p *(2 r-1))
$$

donde $p$ es el nivel de ruido máximo, $r$ es un número aleatorio entre 0 y 1 .

\begin{tabular}{clll}
\hline Casos & $\begin{array}{l}\text { Dominio } \\
L_{x} \times L_{y}\end{array}$ & $\begin{array}{l}\text { Fuentes y } \\
\text { detectores }\end{array}$ & $\begin{array}{l}\text { Sistema a resolver } \\
\text { Incógnitas } \times \text { Ecs. }\end{array}$ \\
\hline 1 & $30 \times 60$ & $60-60$ & $1800 \times 3600$ \\
2 & $40 \times 60$ & $60-60$ & $2400 \times 3600$ \\
3 & $50 \times 60$ & $60-60$ & $3000 \times 3600$ \\
4 & $60 \times 60$ & $60-60$ & $3600 \times 3600$ \\
\hline
\end{tabular}

Tabla 1: Detalle técnico de los casos estudiados.

\section{Caso 1: Dominio espacial de $30 \times 60$}

En este caso hemos considerado un dominio espacial de $30 \times 60$ pixeles, en la que se colocan 60 fuentes y 60 detectores. Se obtiene una imagen original con las medidas de los detectores dentro del problema directo.

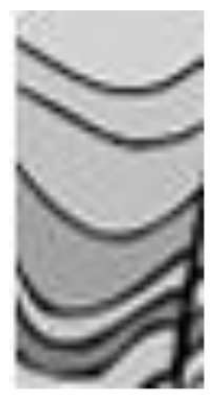

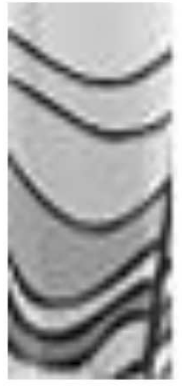

$\lambda=0.0001$

EP:10.11

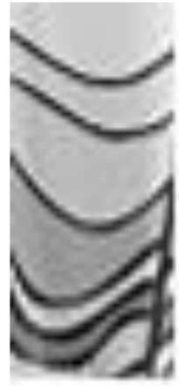

$\lambda=0.001$

$\mathrm{EP}: 10.83$

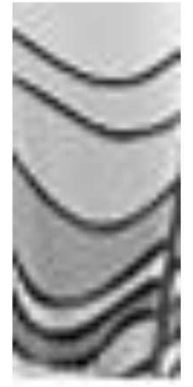

$\lambda=0.01$ EP: 13.16

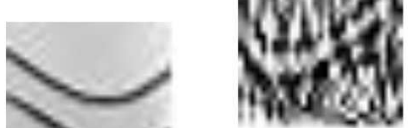

$\lambda=0.1$

$\mathrm{EP}: 45.37$
En la Figura 8 parte superior, se presenta la imagen original sin ruido aleatorio que va a ser reconstruida mediante el método de Levenberg Marquardt con los factores de amortiguamiento $\lambda=0.0001,0.001,0.01$ y 0.1 , cuyos resultados son mostrados en las cuatro imágenes respectivas de la segunda fila de la Figura 8 Debajo de cada imagen de la segunda fila se muestran los errores porcentuales para cada imagen procesada.
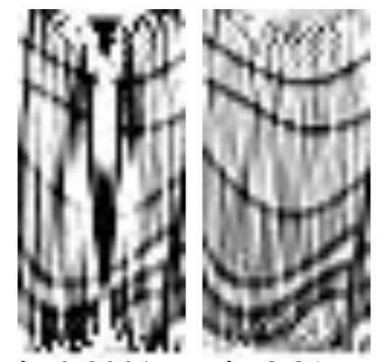

$\lambda=0.0001$

$\lambda=0.01$

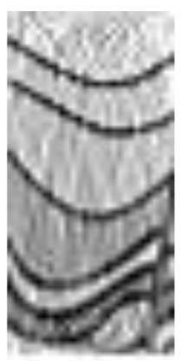

$$
\lambda=0.1
$$

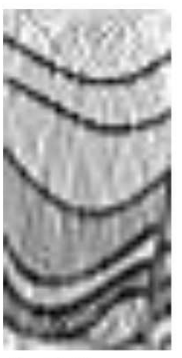

EP:70.75

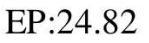

$\mathrm{EP}: 19.16$

$\lambda=0.2$
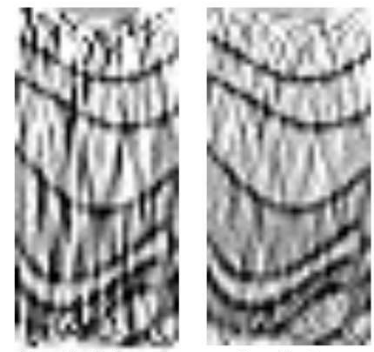

$\lambda=0.01$

$\lambda=0.1$

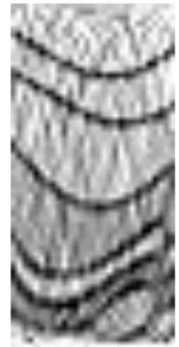

$\lambda=0.2$

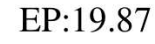

EP:40.18

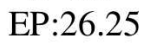

$\mathrm{EP}: 24.94$

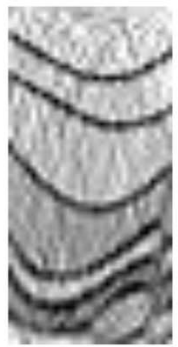

$\lambda=0.5$
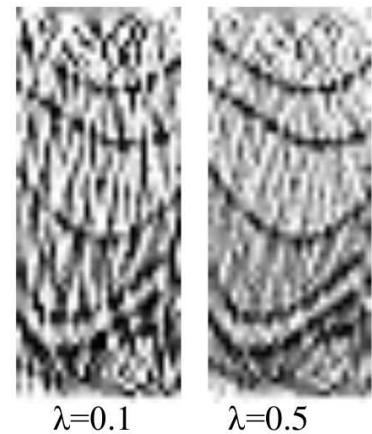

$\lambda=0.5$

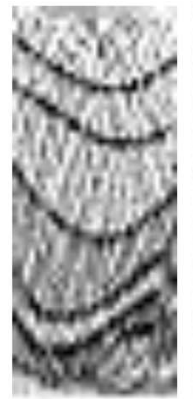

$\lambda=0.8$

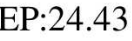

Figura 9: Imágenes reconstruidas con los datos que incorporan ruido aleatorio de $1 \%$, $3 \%$ y $5 \%$, para cada fila de arriba para abajo, respectivamente.

En la Figura 9 se muestran tres filas de imágenes procesadas con ruidos aleatorios en los datos con un porcentaje de $1 \%$ (fila superior), $2 \%$ (fila intermedia) y $5 \%$ (fila inferior). Las imágenes en columnas de izquierda a derecha con ruido aleatorio de $1 \%$ y con los factores de amortiguamiento $\lambda=0.0001,0.01,0.1$ y 0.2 , respectivamente, se muestran en la primera fila. Las imágenes de izquierda a

En la parte inferior se muestran las imágenes reconstruidas por el método Levenberg Marquardt. 
derecha con $\lambda=0.01,0.1,0.2$ y 0.5 con ruido aleatorio de $3 \%$ se muestran en la fila intermedia y las imágenes de izquierda a derecha en la fila inferior de la figura con los factores de amortiguamiento $\lambda=0.1,0.5,0.8$ y 1.0 son mostrados. En la parte inferior de todas las imágenes se muestran además del factor de amortiguamiento empleado, el error porcentual obtenido en la reconstrucción de cada imagen.

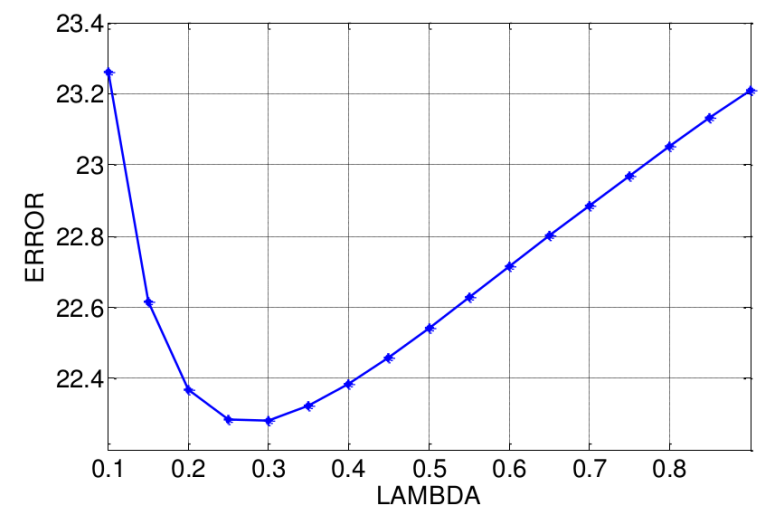

Figura 10: Se muestra el error porcentual al obtener las lentitudes en función de los factores de amortiguamiento $\lambda$ para la reconstrucción de imágenes con datos que incluyen ruido de $1.5 \%$.

Una revisión cualitativa de la calidad de las imágenes de Is Fig 9 nos permite concluir que a mayor nivel del ruido, los valores de los factores de amortiguamiento aumentan y los errores porcentuales en la determinación de las lentitudes disminuye alcanzando un mínimo para un nivel de ruido dado. Para las imágenes reconstruidas con los datos con ruido aleatorio de $1.5 \%$, el error porcentual en la obtención de las lentitudes en función de los factores de amortiguamiento presentan una evolución que se muestra en la Figura 10] y se observa que el error mínimo encontrado para esta configuración espacial de pixeles se encuentra para un valor de $\lambda$ próximo a 0.28 .

\begin{tabular}{llll}
\hline $\begin{array}{l}\text { Nivel } \\
\text { ruido }\end{array}$ & $\lambda_{\text {optimo }}$ & $\begin{array}{l}\text { Error por- } \\
\text { centual }\end{array}$ & $\begin{array}{l}\text { Tiempo } \\
(\mathrm{s})\end{array}$ \\
\hline 0.0 & $10^{-10}$ & 9.70 & 18 \\
0.5 & 0.015 & 15.67 & 18 \\
1.0 & 0.080 & 20.78 & 18 \\
1.5 & 0.250 & 22.28 & 18 \\
2.0 & 0.500 & 24.12 & 18 \\
2.5 & 0.800 & 25.94 & 18 \\
3.0 & 1.000 & 26.60 & 18 \\
\hline
\end{tabular}

Tabla 2: Resumen de las reconstrucciones de imágenes obtenidas por el método de Levenberg Marquardt para un dominio espacial de $30 \times 60$ pixeles.

Este proceso de reconstrucción de imágenes se realiza para diferentes niveles de ruido cuyas figuras no se muestran por motivo de espacio, pero presentamos en la Tabla
2 los factores de amortiguamiento que minimizan los errores porcentuales en la determinanción de las lentitudes y el tiempo de propagación de las ondas sísmicas entre las fuentes y los detectores, todos dependientes del nivel de ruido aleatorio aplicado a los datos.

\section{Caso 2: Dominio espacial de $40 \times 60$}

Consideramos un dominio espacial de $40 \times 60$, con 60 fuentes y 60 detectores. De los datos obtenidos por los detectores obtenemos la imagen original que se muestra en la fila superior de la Fig 11 mientras que las imágenes reconstruidas con el método de Levenberg Marquardt son mostrados de izquierda a derecha y corresponden a los niveles de ruido de $0 \%, 1 \%, 2 \%$ y $3 \%$, mientras que los parámetros de amortiguamiento mínimos correspondientes son $10^{-4}, 0.08,0.4$ y 1.0 , respectivamente. $Y$ los errores porcentuales de determinación de las lentitudes correspondientes para cada imagen son $15.36 \%, 33.65 \%, 35.56 \%$ y $41.58 \%$. Esta figura resume todo el procedimiento que hemos realizado con detalle y que está descrito en el caso anterior.

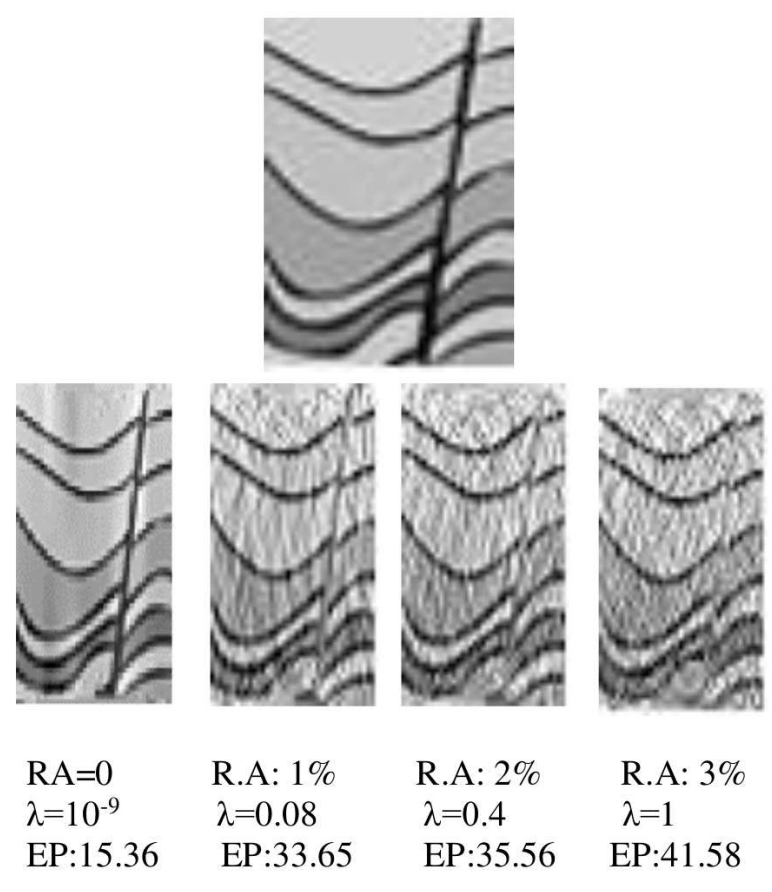

Figura 11: Se muestra la imagen original en la parte superior. En la parte inferior se muestran las imágenes reconstruidas con los factores de amortiguamiento que permite una minimización de los errores porcentuales en la determinación de las lentitudes para diferentes niveles de ruido aleatorio (R.A.).

La Tabla 3 resume la reconstrucción de las imágenes a cuyos datos fueron añadidos diferentes niveles de ruido aleatorio determinando los factores de amortiguamiento que permiten minimizar los errores porcentuales obteni- 
dos en la determinación de las lentitudes y los tiempos de recorrido de las ondas entre las fuentes y los detectores.

\begin{tabular}{llll}
\hline $\begin{array}{l}\text { Nivel de } \\
\text { ruido }\end{array}$ & $\lambda_{\text {optimo }}$ & $\begin{array}{l}\text { Error por- } \\
\text { centual }\end{array}$ & $\begin{array}{l}\text { Tiempo } \\
(\mathrm{s})\end{array}$ \\
\hline 0.0 & $10^{-10}$ & 15.36 & 26 \\
0.5 & 0.015 & 23.83 & 26 \\
1.0 & 0.080 & 33.65 & 26 \\
1.5 & 0.200 & 34.87 & 26 \\
2.0 & 0.400 & 35.56 & 26 \\
2.5 & 0.700 & 39.93 & 26 \\
3.0 & 1.000 & 41.58 & 26 \\
\hline
\end{tabular}

Tabla 3: Resumen de las reconstrucciones de imágenes obtenidas por el método de Levenberg Marquardt para un dominio espacial de $40 \times 60$ pixeles.

\section{Caso 3: Dominio espacial de $50 \times 60$}

Consideramos un dominio espacial de $50 \times 60$ pixeles, con 60 fuentes y 60 detectores. Aplicamos el método de Levenberg Marquardt para el proceso de reconstrucción de las imágenes a cuyos datos originales fueron introducidos ruido aleatorios de diferentes amplitudes. El detalle del proceso es explicado en el caso 1 .

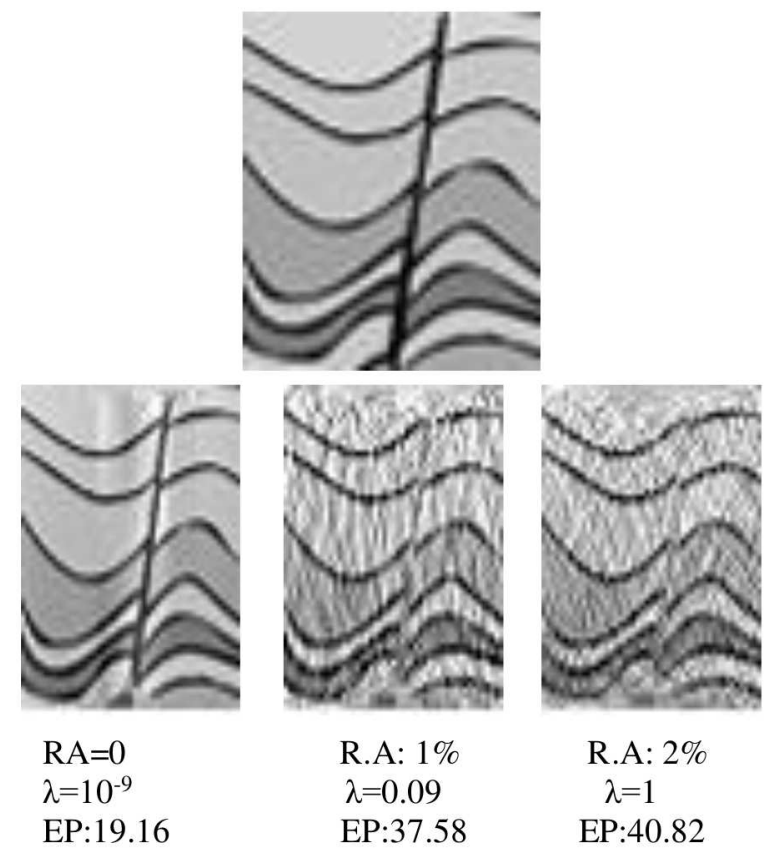

Figura 12: La parte superior muestra la imagen original, mientras que en la parte inferior se muestran las imágenes reconstruidas con los factores de amortiguamiento que minimizan los errores porcentuales en la determinación de las lentitudes para diferentes amplitudes de ruido aleatorio.

La parte superior de la Figura 12 presenta la imagen original, mientras que las tres imágenes mostradas en la parte inferior son las reconstruídas considerando los factores de amortiguamiento $\lambda$ que minimizan los errores porcentuales en la determinación de las lentitudes. Los detalles de los valores de los ruidos aleatorios aplicados, los factores de amortiguamiento aplicados y los errores obtenidos se muestran en la parte inferior de cada imagen.

Un resumen del proceso llevado a cabo para esta configuración espacial de $50 \times 60$ con datos a los se incluyen diferentes niveles de ruido se presentan en la Tabla 4.

\begin{tabular}{llll}
\hline $\begin{array}{l}\text { Nivel de } \\
\text { ruido }\end{array}$ & $\lambda_{\text {optimo }}$ & $\begin{array}{l}\text { Error por- } \\
\text { centual }\end{array}$ & $\begin{array}{l}\text { Tiempo } \\
(\mathrm{s})\end{array}$ \\
\hline 0.0 & $10^{-10}$ & 19.16 & 29 \\
0.5 & 0.015 & 29.70 & 29 \\
1.0 & 0.080 & 37.58 & 29 \\
1.5 & 0.250 & 39.28 & 29 \\
2.0 & 1.000 & 40.82 & 29 \\
\hline
\end{tabular}

Tabla 4: Resumen de las reconstrucciones de imágenes obtenidas por el método de Levenberg Marquardt para un dominio espacial de $50 \times 60$ pixeles.

\section{Caso 4: Dominio espacial de $60 \times 60$}

El dominio espacial considerado es de $60 \times 60$ pixeles, con 60 fuentes y 60 detectores, los que nos proporciona un sistema de ecuaciones de igual número de incógnitas y ecuaciones.

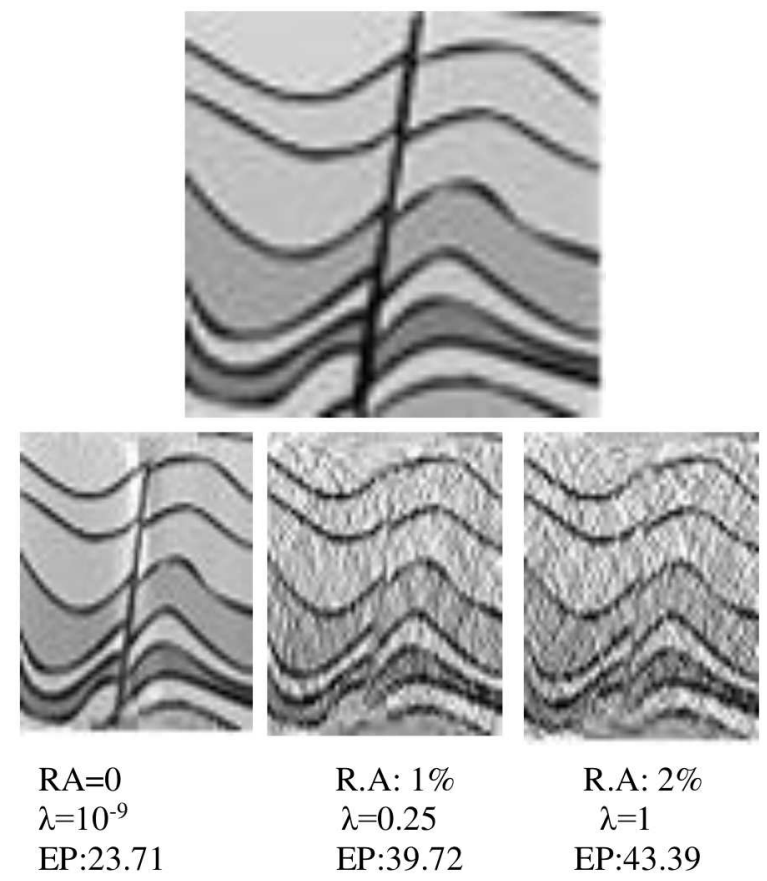

Figura 13: En la parte superior se muestra la imagen original. En la parte inferior se muestran tres imágenes reconstruidas con el método de Levenberg Marquardt para diferentes amplitudes de ruido aleatorio introducidos a los datos de la imagen original. 
La Figura 13 presenta en la parte superior la imagen original de un dominio descrito en el párrafo anterior, mientras que en la parte inferior se muestran tres imágenes reconstruidas mediante el método de Levenberg Marquardt con los factores de amortiguamiento que minimizan los errores porcentuales en la obtención de las lentitudes para los diferentes niveles de ruido aleatorio que se introducen en los datos de la imagen original. La Tabla 5 presenta un resumen de todos los procesos realizados con los datos de la Figura 13.

\begin{tabular}{llll}
\hline $\begin{array}{l}\text { Nivel de } \\
\text { ruido }\end{array}$ & $\lambda_{\text {optimo }}$ & $\begin{array}{l}\text { Error por- } \\
\text { centual }\end{array}$ & $\begin{array}{l}\text { Tiempo } \\
(\mathrm{s})\end{array}$ \\
\hline 0.0 & $10^{-10}$ & 23.71 & 37 \\
0.5 & 0.07 & 35.58 & 37 \\
1.0 & 0.25 & 39.72 & 37 \\
1.5 & 0.98 & 42.10 & 37 \\
2.0 & 1.00 & 43.39 & 37 \\
\hline
\end{tabular}

Tabla 5: Resumen de las reconstrucciones de imágenes obtenidas por el método de Levenberg Marquardt para un dominio espacial de $60 \times 60$ pixeles.

Cabe mencionar que hemos realizado la reconstrucción de imágenes usando el método de mínimos cuadrados y el de Newton, pero no muestran un resultado de reconstrucción significativo. Los resultados obtenidos para los casos 1-4 con el método de Levenberg Marquardt iterativo son similares. Sin embargo en el proceso iterativo es necesario considerar un valor inicial adecuado, y tener un criterio de parada para el número de iteraciones.
En este trabajo se consideró 0.5 como el valor de los todos elementos del vector de la lentitud inicial, $S^{0}$, y el número de iteraciones óptimo en la mayoría de los casos fue de 1.

\section{Conclusiones}

El método de Levenberg Marquardt, se puede emplear en tomografía de pozo a pozo, considerando el factor de amortiguamiento lambda adecuado para el nivel de ruido existente. Notamos que los sistemas de ecuaciones que doblan el número de ecuaciones al de incógnitas soportan más ruido aleatorio, como en el caso 1 , a medida que la relación del número de ecuaciones e incógnitas disminuye, el sistema de ecuaciones soporta menos ruido, casos 3 y 4. Los métodos de mínimos cuadrados y de Newton, no logran reconstruir las imágenes de estos casos. El método de Levenberg Marquardt iterativo ofrece similares resultados que el Método de Levenberg Marquardt matricial, sin embargo hay que notar que en el proceso iterativo es necesario encontrar un valor inicial y determinar el número de iteraciones adecuado. En este trabajo se empleó el valor 0.5 para los todos los elementos de la lentitud, $S^{0}$, y el número iteraciones óptimo fue 1.

\section{Agradecimientos}

Los autores agradecen al profesor J. P. Zubelli del IMPA, Brasil, por las sugerencias que permitieron el desarrollo de este trabajo.

\section{Referencias}

[1] Gerard T. Schuster; Basics of exploration seismology and tomography, Mathematical Geophysics Summer School Lectures, Stanford (1998).

[2] K. A. Dines y R. J. Lytke; Proc. IEEE 67, 1065 (1979).

[3] N. D. Bregman, R. C. Bailey y C. H. Chapman; Geophysics 54, 200 (1989).

[4] G. Briao; Tomografía Sísmica por Tempo de Percurso: Modelagem, Métodos Numéricos e Implementação, dissertação de Mestrado, Instituto Nacional de Matemática Pura e Aplicada, Brasil (2005).

[5] M. Flores; Procesado de sísmica de reflexión superficial; acceso en línea, 15 de Febrero de 2012.

[6] J. Berryman; Phys. Rev. Lett. 62(25), 2953 (1989).
[7] J. Berryman, Lecture notes on nonlinear inversión and tomography. I. Borehole Seismic Tomography. http://sepwww.stanford.edu/sep/berryman /NOTES/lecture_notes.html

[8] M. Sánchez, Tomografia lineal con residuos temporales bajo la península Ibérica, Tesis doctoral, Universidad complutense de Madrid (1995).

[9] S. Souza; Uma Classe de Métodos Interior-proximais com Métrica Variável para Problemas Convexos e um Algoritmo Interior-proximal com Distância de Bregman para Otimização Quase-convexa. Tesis de Doctorado, Ingenieria de Sistemas, COPPE -UFRJ (2007).

[10] D. P. Bertsekas, Non Linear Programming, Athenea Press, Belmont (1995).

[11] F. Natterer y F. Wubbeling; Mathematical methods image reconstruction, segunda edición, SIAM, Philadelphia (2001). 